\section{Angle closure glaucoma associated with ectopia lentis in a patient with Sturge-Weber syndrome}

Eye (2012) 26, 342; doi:10.1038/eye.2011.304
DB Moore, SD Reck and PP Chen

The authors would like to apologise for this error.

Correction to: Eye 25, 1235-1236; doi:10.1038/

sj.eye.2011.101; published online 6 May 2011

Since the online publication of this paper the authors have noticed that they omitted to acknowledge support received in the form of a departmental grant from Research to Prevent Blindness, Inc., New York, NY, USA. 